\title{
Electromyographic patterns of the rat hindlimb in response to muscle stretch after spinal cord injury
}

\author{
Anastasia V. Keller ${ }^{1} \cdot$ Kathlene M. Rees ${ }^{2} \cdot$ Erik J. Seibt $^{2} \cdot$ B. Danni Wood ${ }^{2} \cdot$ Abigail D. Wade $^{2} \cdot$ Johnny Morehouse $^{2} \cdot$ \\ Alice Shum-Siu ${ }^{2} \cdot$ David S. K. Magnuson $\mathbb{D I}^{1,2,3}$
}

Received: 5 September 2017 / Revised: 17 January 2018 / Accepted: 21 January 2018 / Published online: 19 February 2018

(c) International Spinal Cord Society 2018

\begin{abstract}
Study design Experimental Study.

Objectives To characterize the specific hindlimb electromyographic (EMG) patterns in response to muscle stretch and to measure the applied forces during stretching in the rat model of moderate SCI.

Setting Kentucky Spinal Cord Injury Research Center, Louisville, KY, USA.

Methods Female Sprague Dawley rats $(n=4)$ were instrumented for telemetry-based EMG recording (right rectus femoris and biceps femoris) and received a moderate T10 spinal cord injury (SCI). The major hindlimb muscle groups were stretched using our clinically modeled protocol. The EMG responses were recorded biweekly for 8 weeks. The forces applied during stretching were measured using a custom-designed glove. Locomotor function was assessed using the BBB Open Field Locomotor Scale, 3D kinematics and gait analysis.

Results Three main EMG patterns in response to stretch were identified: clonic-like, air-stepping, and spasms. Torques applied during stretching ranged from $0.4-8 \mathrm{~N} \bullet \mathrm{cm}$, and with the exception of the quadriceps, did not change significantly over the weeks of stretching. Two stretching sessions a week did not result in a significant disruption to locomotor function. Conclusions Stretching evokes EMG patterns in rats similar to those reported in humans including clonus and spasms. The torques used during stretching are comparable, based on the ratio of torque to body weight, to the few previously published studies that measured the forces and/or torques applied by physical therapists when stretching patients. Future studies are warranted to fully explore the impact of muscle stretch on spinal cord function after injury.
\end{abstract}

Sponsorship DoD, KSCHIRT, NIH.

\section{Introduction}

Stretching remains a leading therapy for the treatment of muscle contractures and spasticity, two common complications after a spinal cord injury (SCI) $[1,2]$. Moreover, stretching is a rehabilitation strategy often employed acutely post-SCI in an effort to prevent the development of muscle

David S. K. Magnuson

dsmagn01@louisville.edu

1 Departments of Physiology, University of Louisville, Louisville, USA

2 Departments of Neurological Surgery, University of Louisville, Louisville, USA

3 Departments of Anatomical Sciences and Neurobiology, University of Louisville, Louisville, USA contractures because they are much harder to treat once established [3]. The rationale for using stretching in the rehabilitation of soft tissue contractures arose initially from animal studies that showed it to be effective at maintaining range of motion (ROM) during limb immobilization in otherwise intact mice [4]. However, stretching for the treatment or prevention of contractures in human subjects with SCI does not have the same efficacy [5]. We determined recently that a daily protocol of either static [6] or dynamic muscle stretch [7] significantly disrupts hindlimb locomotor function in rats with SCI. We documented limb movements during and immediately post-stretch that ranged from low frequency air-stepping to high frequency, cloniclike "vibrations" [7]. The mechanisms underlying the stretch-induced loss of locomotor function are not known but based on our previous findings it is dependent on torques sufficient to achieve a maximum end range-of-motion, but do not involve overt muscle damage [6,7]. It is well 
documented that in healthy subjects static stretching decreases strength and maximum performance [8-11]. Avela et al. [12] have shown that repeated and prolonged stretching results in decreased electromyographic (EMG) amplitude during maximum voluntary contraction, suggesting a central mechanism. Interestingly, Cramer et al. [13], observed strength loss not only in the stretched limb but also in the contralateral non-stretched limb, further implicating a spinal circuitry-based mechanism. Given these observations and widespread use of stretching in the rehabilitation/physical therapy of human subjects with SCI [3, 14] it is important to establish if the negative effects of stretching on locomotor function in rats with SCI are clinically relevant. Therefore, we designed and performed an experiment using tools that would allow direct comparison with human studies, such as electromyography, force and torque measurements and limb kinematics during stretching. EMG patterns represent the response of the spinal cord to the afferent input generated by stretching and thus can serve as a translational cue: if responses are similar for rats and humans, then it is more likely that the impact of stretching on motor circuitry will also be similar. Force and torque measurements will provide context for the EMG responses and will help establish the parameters for future clinical studies on stretching after SCI.

\section{Methods}

\section{Experimental design}

This small ' $n$ ' preliminary study sought to document the patterns of EMG activity elicited by muscle-stretch after SCI and to gain some insight into the relationship between applied torque, EMG response, and time post-injury without the loss of locomotor function induced by daily stretching in this model. The three-phase experimental design was influenced by the high cost of EMG transmitters and the labor-intensive nature of the data collection and analysis. The first of three phases involved 6 weeks where we chose to stretch the un-implanted hindlimb only, due to the risk of disturbing the EMG wires in the implanted limb. We stretched only twice a week to avoid inducing a drop in locomotor function [6] and to focus on how applied torque and elicited EMG patterns changed over time. The second phase involved 2 weeks of daily stretches (full protocol) of the un-instrument limb plus the ankle flexors and extensors of the instrumented limb which allowed us to observe direct stretch-induced responses while still not risking disruption of the EMG leads in the flexor and extensor muscles of the knee. The final phase involved one terminal stretching session where the full protocol was applied to both hindlimbs.
Animals, EMG transmitter instrumentation, and SCI

Four adult female Sprague Dawley rats, 225-250 g (Harlan, Indianapolis, IN), were used for this experiment. All procedures involving animals were approved by the Institutional Animal Care and Use Committee at the University of Louisville. Animals were housed in pairs in standard cages with alpha-dri bedding and a 12/12 light/dark cycle. After one week of standard gentling procedures and baseline assessments the animals underwent an initial surgery for instrumentation with a telemetry-based 2 lead EMG transmitter (F20-EET, Data Sciences International ${ }^{\circledR}$, St. Paul, $\mathrm{MN})$. The animals were anesthetized with a ketamine cocktail and a surgical level of anesthesia was confirmed by the absence of paw withdrawal reflexes to strong foot pinch. The upper back of each animal was shaved and cleaned and a small skin incision was made between the shoulder blades to allow placement of the transmitter. The wire electrodes of the transmitter were tunneled subcutaneously to the right hindlimb by separating the skin from the muscle layer using blunt dissection. A small incision was made over the thigh of the animal allowing the electrodes to be inserted into and through the main belly of the rectus femoris and biceps femoris muscles. Sutures were placed to hold the electrodes in place. The incisions were closed with sutures and animals were allowed to recover, receiving our standard postoperative care with antibiotics (gentamicin sulfate for 7 days $(20 \mathrm{mg} / \mathrm{kg})$ ) and pain management (buprenorphine $0.03 \mathrm{mg} / \mathrm{kg}$ twice a day for 3 days). Two weeks later the animals underwent a second surgery and received a moderate spinal cord contusion injury at thoracic level T10 $(12.5 \mathrm{~g} / \mathrm{cm}, \mathrm{NYU}$ Mascis Impactor) as previously described [15]. The animals were then allowed to recover for 2 weeks prior to the initiation of stretching.

\section{Stretching and recording procedures}

In this study we used six stretches (1 min static hold) of the major hindlimb muscle groups (knee and ankle flexors and extensors plus hip adductors and abductors) according to our previously described stretching protocol that was modeled after local clinical practices and with the assistance of experienced physical therapists [16, 17]. Animals were moved in their home cages to the laboratory each day of stretching and assessment. EMG data were acquired through the DataQuest Acquisition hardware (Data Sciences ${ }^{\circledR}$ International, St. Paul, MN) and PONEMAH ${ }^{\circledR}$ 5.0 software package (sampled at $1000 \mathrm{~Hz}$ ). The data were exported for further analysis to LabChart (ADInstuments Colorado Springs, CO). Within the DSI system, the receiver and the transmitter communicate at a set frequency of 455 $\mathrm{kHz}$. This reduces the possibility of signal contamination with non-specific frequencies from surrounding electronics. 


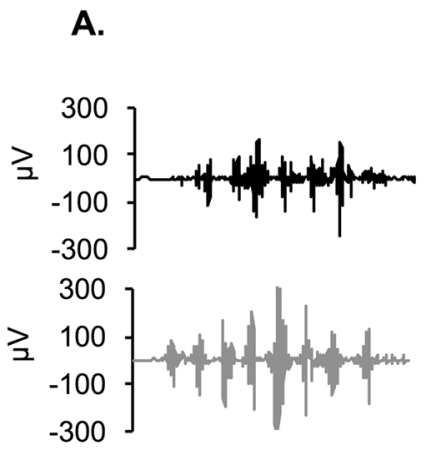

B. Air stepping
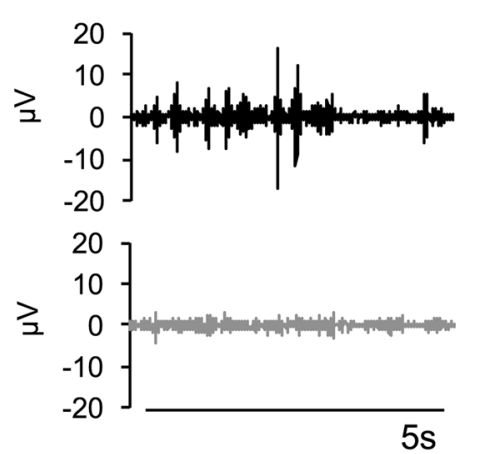

Fig. 1 Typical EMG responses to stretch. The top and bottom traces show the responses of rectus femoris and biceps femoris respectively of the contralateral limb. Panel a shows one example of clonic-like contractions from each animal. Other common responses included air stepping with predominantly extensor activity (b) and spasms (c).

During stretching and recording, the animals were wrapped in a terry-cloth towel as described previously and held ventral side up directly on the receiver ensuring a strong signal for data transfer from the transmitter to the receiver. These features, in addition to the isolation of the preamplifier, transmitter and the EMG leads within the animal ensure a high quality EMG signal with a very high signal to noise ratio $(>100)$ and with no noticeable $60 \mathrm{~Hz}$ contamination. The raw EMG signal was band-pass filtered at $50-2000 \mathrm{~Hz}$ to remove movement artifacts. All clonic-like responses were analyzed for peak amplitude and frequency using rectified and smoothed EMG traces and LabChart's peak analysis feature. Videos of the implanted limb were linked to EMG recordings and each analyzed clonic-like response was confirmed visually in the video.

\section{Forces applied during stretching}

The forces applied during stretching were measured using a custom designed glove fitted with FlexiForce A301 sensors connected to a USB-6210 data acquisition system operated by LabView software. Force sensors were calibrated to standardized weights of 105,305 , and $505 \mathrm{~g}$ prior to each stretching session. Force sensor signals were sampled at $1000 \mathrm{~Hz}$. Sensors for the thumb (TS) and index finger (IS)
Panel d shows the position of the rat (dorsal recumbancy) during the stretching of the Tibialis Anterior muscle. Symbols 1-5 represent the toe, ankle, knee, hip, and iliac crest for the purposes of kinematic analysis for calculation of torque. The force sensor is positioned between the thumb and dorsal aspect of the foot near the toe

were used to measure forces during the four stretches. They were placed as follows: for stretch of the Tibialis Anterior (ankle flexor) TS was placed on the dorsal portion of the foot and IS on the heel; for stretch of Triceps Surae we used only one sensor (TS), placed on the mid plantar portion of the foot; for quadriceps TS was placed on top of the knee (partially flexed for this stretch), while IS was placed at the mid dorsal portion of the foot; and for the hamstring stretch TS was placed at the heel and IS on the thigh slightly superior to the knee (extended for this stretch). The forces were not measured during hip abduction and adduction stretches due to difficulty of locating precise points of force application. The positions of the toe, ankle, knee, hip, and iliac crest, along with the sensors were determined using MaxTraq kinematic software (Innovision Systems, Inc., Columbiaville, MI). The distances between the joints and sensors allowed the applied torque to be calculated (torque $=$ force $\times$ distance $(\mathrm{cm}) \times 9.8\left(\mathrm{~m} / \mathrm{s}^{2}\right) / 1000(\mathrm{~g} / \mathrm{kg})$, where 9.8 is the gravitational acceleration constant).

\section{Locomotor function assessments}

Locomotor function was assessed using 3D kinematics and gait analysis based on paw placement order. Horizontal and ventral view recordings were made pre- (early morning) and 
Table 1 Quantification of amplitude and frequency of clonic-like EMG responses to stretch in the contralateral limb at week 2 and 8 , and ipsilateral limbs at week 8

A.

EMG amplitude $(\mu \mathrm{V})$

\begin{tabular}{|c|c|c|c|c|c|c|}
\hline \multirow[b]{2}{*}{ Stretch } & \multicolumn{2}{|l|}{ Week 2} & \multicolumn{2}{|c|}{ W8 contralateral limb } & \multicolumn{2}{|c|}{ W8 ipsilateral limb } \\
\hline & $\begin{array}{l}\text { Rectus } \\
\text { Femoris }\end{array}$ & $\begin{array}{l}\text { Biceps } \\
\text { femoris }\end{array}$ & $\begin{array}{l}\text { Rectus } \\
\text { femoris }\end{array}$ & $\begin{array}{l}\text { Biceps } \\
\text { femoris }\end{array}$ & $\begin{array}{l}\text { Rectus } \\
\text { femoris }\end{array}$ & $\begin{array}{l}\text { Biceps } \\
\text { femoris }\end{array}$ \\
\hline TA & $17.4 \pm 12.74$ & $13.53 \pm 10.45$ & $45.22 \pm 24.24$ & $33.10 \pm 17.97$ & $55.76 \pm 19.73$ & $25.97 \pm 21.84$ \\
\hline Gastroc & ${ }^{\mathrm{a}} 26.36 \pm 16.10$ & $\begin{array}{l}{ }^{\mathrm{a}} 19.49 \pm \\
23.20\end{array}$ & $\begin{array}{l}\mathrm{b}_{23.95} \pm \\
21.60\end{array}$ & $\begin{array}{l}\mathrm{b}_{20.98} \pm \\
15.65\end{array}$ & $\begin{array}{l}{ }^{\mathrm{a}} 28.67 \pm \\
23.76\end{array}$ & ${ }^{\mathrm{a}} 9.60 \pm 6.06$ \\
\hline RF & ${ }^{\mathrm{a}} 15.85 \pm 5.49$ & ${ }^{\mathrm{a}} 6.75 \pm 3.20$ & $43.85 \pm 16.84$ & $30.64 \pm 24.59$ & $81.83 \pm 37.78$ & $59.96 \pm 37.95$ \\
\hline Hamstring & $9.13 \pm 3.31$ & $5.38 \pm 3.81$ & $\mathrm{a} 22.00 \pm 2.17$ & $\begin{array}{l}\text { a } 41.07 \pm \\
37.71\end{array}$ & $38.79 \pm 23.33$ & $23.97 \pm 21.75$ \\
\hline
\end{tabular}

B.

EMG frequency $(\mathrm{Hz})$

\begin{tabular}{|c|c|c|c|c|c|c|}
\hline \multirow[b]{2}{*}{ Stretch } & \multicolumn{2}{|l|}{ Week 2} & \multicolumn{2}{|c|}{ W8 contralateral limb } & \multicolumn{2}{|c|}{ W8 ipsilateral limb } \\
\hline & $\begin{array}{l}\text { Rectus } \\
\text { femoris }\end{array}$ & $\begin{array}{l}\text { Biceps } \\
\text { femoris }\end{array}$ & $\begin{array}{l}\text { Rectus } \\
\text { femoris }\end{array}$ & $\begin{array}{l}\text { Biceps } \\
\text { femoris }\end{array}$ & $\begin{array}{l}\text { Rectus } \\
\text { femoris }\end{array}$ & $\begin{array}{l}\text { Biceps } \\
\text { femoris }\end{array}$ \\
\hline TA & $5 \pm 0.82$ & $4.75 \pm 0.96$ & $6.5 \pm 0.58$ & $6.5 \pm 1$ & $6 \pm 0.82$ & $6 \pm 1.63$ \\
\hline Gastroc & ${ }^{\mathrm{a}} 4 \pm 1.0$ & ${ }^{\mathrm{a}} 4.33 \pm 0.58$ & $\mathrm{~b}_{5}$ & $\mathrm{~b}_{6}$ & ${ }^{a} 5 \pm 1.0$ & ${ }^{\mathrm{a}} 5.67 \pm 1.52$ \\
\hline $\mathrm{RF}$ & ${ }^{\mathrm{a}} 4.3 \pm 0.58$ & ${ }^{\mathrm{a}} 4.3 \pm 0.58$ & $6.25 \pm 0.95$ & $6.75 \pm 1.5$ & $7 \pm 1.82$ & $7.5 \pm 1.91$ \\
\hline Hamstring & $4.75 \pm 0.98$ & $4.75 \pm 1.25$ & ${ }^{\mathrm{a}} 4.33 \pm 0.58$ & ${ }^{a} 5 \pm 1$ & $4.75 \pm 1.71$ & $5 \pm 0.82$ \\
\hline
\end{tabular}

Data is shown as mean \pm standard deviation

Although there were no statistically significant differences due to small sample size and high variability, there was a trend towards increased amplitude and frequency of clonic-like contractions from week 2 to 8 , most pronounced in responss to quadriceps and Tibialis Anterior stretch. ${ }^{\mathrm{a}} n=3$

${ }^{\mathrm{b}} n=2$, if clonus was not observed during that stretch at that time point in the animal; otherwise, $n=4$

post-stretching (early afternoon) for each session. As previously described $[15,18]$ we used a 3 segment 2 angle model of the hindlimb for kinematics with the iliac cresthip-ankle (IHA) and hip-ankle-toe (HAT) angles representing the proximal (hip and knee) and distal (knee and ankle) aspects of the limb. This model avoids the significant inaccuracies associated with skin slippage about the knee during stepping. These angles were measured using MaxTraq software with final excursions determined using a custom designed excel macro. Gait was assessed using the Regularity Index, calculated as the number of correctly patterned plantar steps over the total number of steps [18]. BBB open field locomotor assessments [19] were done once a week on Fridays (afternoon).

\section{Sacrifice and tissue histology}

Animals were sacrificed using a ketamine $(50 \mathrm{mg} / \mathrm{kg}) /$ xylazine $(0.024 \mathrm{mg} / \mathrm{kg}) /$ acepromazine $(0.005 \mathrm{mg} / \mathrm{kg})$ cocktail and transcardially perfused [20] with phosphate buffer. The spinal cord was dissected out and postfixed in 4\% PFA overnight and cryoproteced in 30\% sucrose for at least 4 days. The injury level (T10) was confirmed visually using a dissecting microscope, and the injury epicenter was blocked in tissue freezing medium. Transverse sections were cut at $30 \mu \mathrm{m}$ on a cryostat for histological assessment of spared white matter (SWM) at the SCI epicenter. The sections were stained with eriochrome cyanine and imaged at $4 \mathrm{X}$ using a light microscope. Dark blue compact white matter was traced and measured in ImageJ and the section with the lowest percentage of SWM was determined to be the epicenter [21].

Data are shown as mean \pm standard deviation. The outcome measures were analyzed for changes over time using RM ANOVA, followed by a Bonferroni post hoc $t$-test. One-sample $t$-test was used to compare regularity index values between baseline (100\%) and all time points postinjury.

We certify that all applicable institutional and governmental regulations concerning the ethical use of animals were followed during the course of this research. 
Fig. 2 Quantification of weekly forces during the four hindlimb stretches. The recorded forces, expressed as torque $(\mathrm{N} \bullet \mathrm{cm})$, remained consistent through the weeks of stretching for Gastrocnemius (b), Hamstring (d), and Tibialis Anterior (a), except at week 7 , when forces were significantly lower as compared to week 2 , ${ }^{*} p<0.05$. The torque gradually increased with weeks post-SCI for the Quadriceps stretch (c) and were significantly greater at weeks 5,6 , and 8 as compared to week $2, * p<0.05$
A.

Tibialis Anterior Stretch

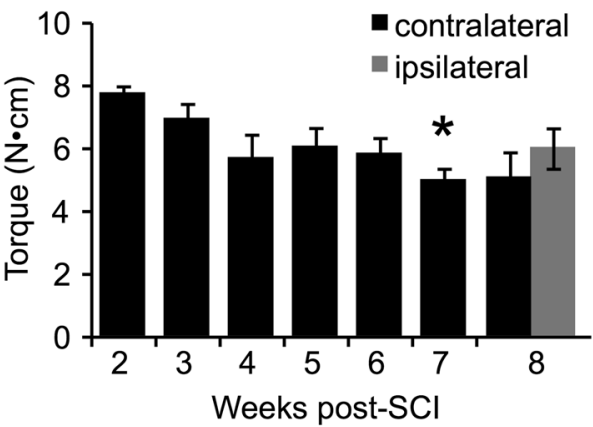

C.

Quadriceps Stretch

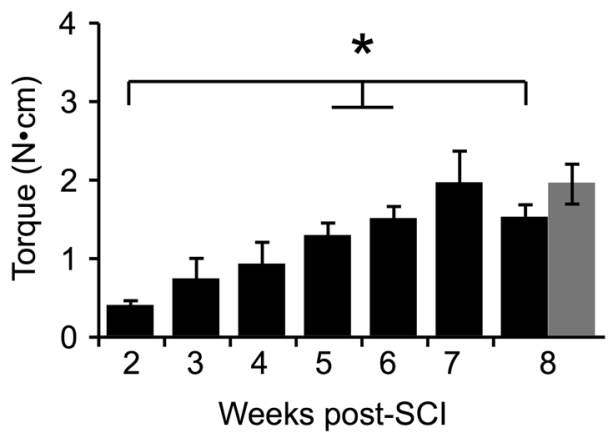

B.

Gastrocnemius Stretch

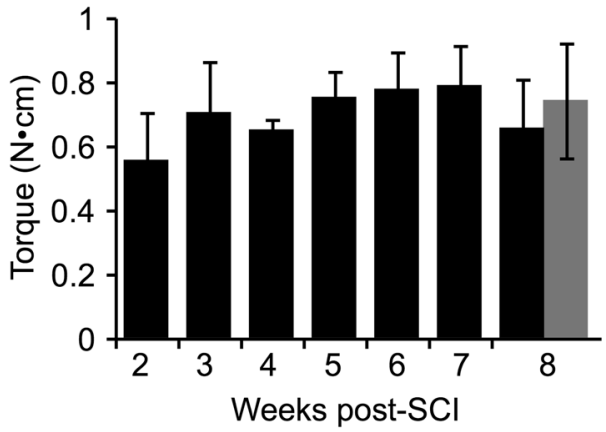

D.

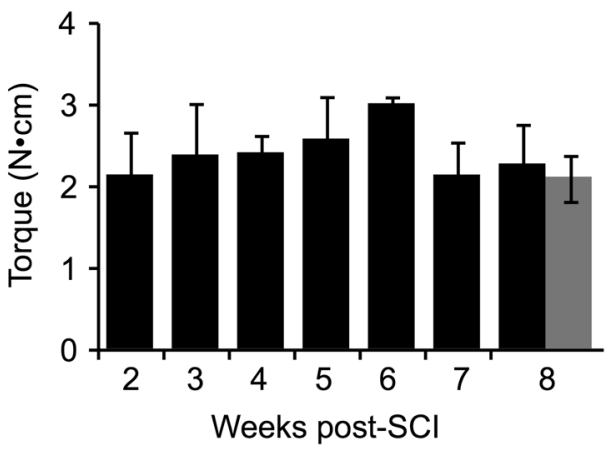

\section{Results}

\section{EMG responses to stretch}

Figure 1 shows representative EMG responses to stretch applied to the contralateral limb. Clonic-like responses (3-9 Hz) included bursts and interburst periods of quiescence with a consistent cycle time and frequencies of 3-9 $\mathrm{Hz}$ ). Air-stepping involved a less defined pattern and movements with frequencies under $3 \mathrm{~Hz}$, whereas spasms were highly irregular bursts with co-activation. Clonic-like responses and spasms were also present in the ipsilateral limb when it was stretched at week 8 , but air stepping could not be observed because the limb was held in a stretched position. These three responses were observed in all animals and are consistent with our previous observations [7, 16]. The responses occur while the limbs are being positioned (pre-stretch) and early in the stretch period but then typically decline while the limb is maintained in the stretched position. Response amplitude and the frequency of cloniclike contractions were quantified for weeks 2 to 8 (weeks 2 and 8 shown in Table 1). Although there were no significant differences over time there was an overall trend of increased amplitude and frequency in clonic-like responses from week 2 to week 8 during most stretches in both contralateral and ipsilateral limbs. Clonic-like contractions were particularly robust during quadriceps and tibialis anterior stretches.

\section{Torques applied during stretching}

The torques applied to achieve the desired end range-ofmotion varied dramatically for the four muscles stretched, with the gastrocnemius requiring about $0.8 \mathrm{~N} \cdot \mathrm{cm}$ and the tibialis anterior requiring about $6 \mathrm{~N} \bullet \mathrm{cm}$ (Fig. 2). Overall, the required torques were consistent over time for the Gastrocnemius (B), Hamstring (D), and TA stretches (A) with the exception of week 7 for the TA. However the torques required for quadriceps stretches gradually increased over time and were significantly greater at weeks 5,6 , and 8 as compared to week 2 (C).

\section{Locomotor function}

Figure 3 shows the weekly BBB scores and subscores (A), regularity index (B) and joint excursions for the hip-ankletoe (C) and iliac crest-hip-ankle (D) angles. All the animals had BBB scores of 21 both pre- and post-implantation of the EMG wires and telemetry transmitter. SCI resulted in significant disruptions to locomotor function, however by week 2 injury the animals were able to generate consistent weight supported stepping (BBB > 10). Gait, assessed using the Regularity Index, was significantly impaired for the first 5 weeks post-SCI as compared to baseline (Fig. 3b). The excursion of the HAT angle, representing the ankle and knee, significantly increased compared to baseline (Fig. 3c), 
Fig. 3 BBB Open Field Locomotor Scores, 3D kinematics and gait analysis of the animals after SCI and during stretching. a Shown are the BBB scores and subscores.

Stretching of one limb twice a week for 6 weeks did not alter the usual improvement seen in BBB scores and subscores. Daily stretching (all six stretches of the non-implanted limb and two ankle stretches of the implanted limb) initiated at week 7 induced a drop in BBB subscore from 4 to 2 , due to changes in toe clearance and paw rotation, that was not statistically significant. b RI was significantly reduced at weeks 2,3 and 4 compared to baseline $(* p<$ $0.05)$. There were no statistically significant differences in pre- and post- stretch RI (regularity index) except at week $6(* p<0.05)$. After SCI the Hip-Ankle-Toe (HAT) excursions were significantly increased $(\mathbf{c})(* p<0.05)$ while the Iliac Crest-Hip-Ankle (IHA) excursions were significantly decreased for the duration of the study (d) $(* p<0.05)$. Pre- and post-stretch HAT and IHA excursions were not different (c, d; assessed using 3D kinematics)

\section{A.}

Open Field Locomotor Scale

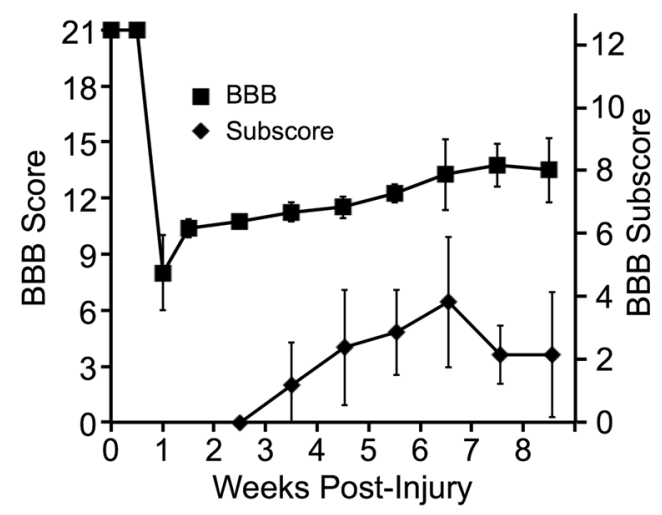

C.

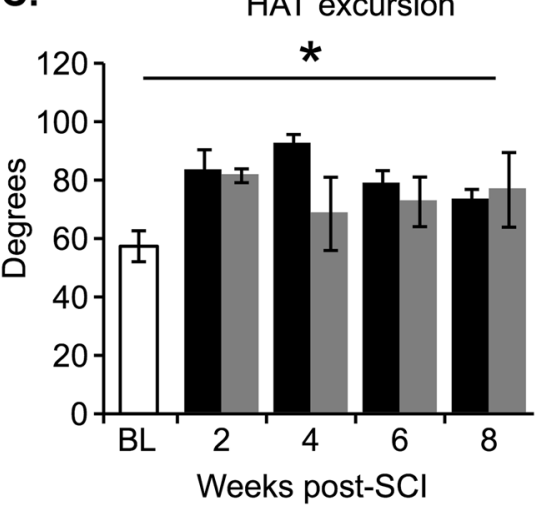

B.

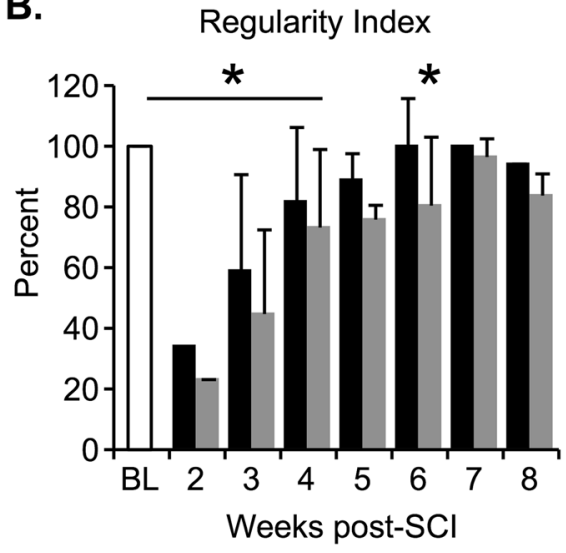

D.

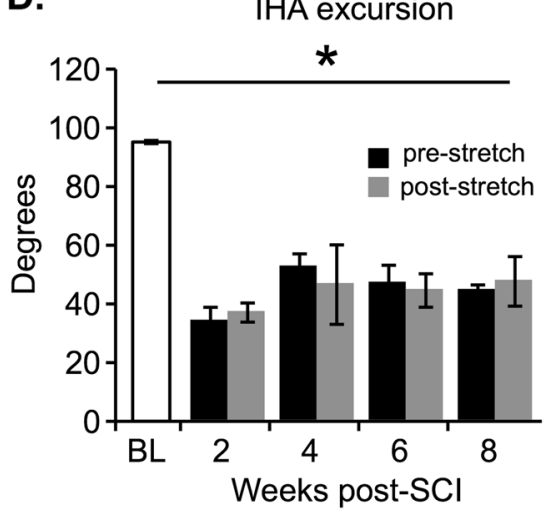

while excursions of the IHA angle, representing the hip and knee, significantly decreased (Fig. 3d). Stretching one limb twice a week had no obvious effect on the locomotor function of the animals over the initial 6 weeks; the profile of recovery was similar to previously published control groups $[6,7,16]$. During weeks 7 and 8 when we began stretching the non-implanted limb daily and added ankle stretches of the implanted limb, BBB scores still remained stable, however, the BBB subscores that assess fine aspects of locomotion including paw position and toe clearance, dropped from an average of 4 to 2.2 during weeks 7 and 8 (Fig. 3a).

\section{Discussion}

Previously $[6,7,16]$ we observed that stretching hindlimb muscles after either moderate or moderately-severe thoracic spinal cord injuries induced motor responses in both the stretched (ipsilateral) and unstretched (contralateral) hindlimbs. These responses were noted and classified as air stepping, spasms and kicking. In the current experiment we determined that the "kicking" responses have a robust EMG burst pattern occurring at $3-9 \mathrm{~Hz}$ that is similar in appearance and frequency to clonus in humans [22]. We observed these clonic-like patterns in response to stretch in both contralateral and ipsilateral limbs. Although clonus in humans with neurological impairments is most frequently seen in the stretched limb one study reported clonus also in the contralateral leg in SCI patients [23]. In our current study the clonic-like responses could be evoked at 2 weeks post-SCI, when we began stretching, however their prevalence, amplitude, and frequency all increased out to 8 weeks. These increases were not statistically significant due to the small sample size and inherent EMG variability. Clonic-like responses were most often observed simultaneously in both recorded muscles, the knee flexor (biceps femoris) and knee extensor (rectus femoris), appearing as co-contractions, although less frequently we also observed clonic-like responses in one but not the other muscle. Co-contractions have also been reported during clonus in human subjects with SCI. Clonus in SCI subjects can occur in response to stretch or other cutaneous inputs and can significantly interfere with the activities of daily living [24]. We did not observe spontaneously occurring clonic-like contractions while the animals were in their cages or during kinematic and gait analysis, although spasms are sometimes observed during open field locomotor assessments. In general, it has been difficult to create a clinically relevant model of spasticity because animals with incomplete 
injuries develop only mild forms of hypereflexia that is difficult to detect and quantify without using velocitydependent measures [25, 26]. However, animals with complete transections at the sacral spinal cord level develop spasticity and tail clonus [27]. More recently, in a study by van Gorp et al. [28], animals exhibited bilateral kicking, suggested to be clonus, in response to nociceptive mechanical stimuli applied to the unloaded hindpaw. In the present study we demonstrate that achieving end range-ofmotion during stretching involves substantial torque equivalent to a $300 \mathrm{~g}$ force ( $\geq$ body weight) applied at $1 \mathrm{~cm}$, thus it is possible that the clonic-like responses are the result of nociceptive signaling. Although clonus in humans is thought to be induced by the activation of Ia afferents [29], a study of human subjects with SCI showed that myoclonus could no longer be elicited when any underlying pathology in the spinal nerve roots, hip joint, or thigh muscles was resolved [30]. Presumably, nociceptive afferent activation subsequent to peripheral tissue damage in these patients triggered clonus or indirectly contributed to the expression of clonus by increasing the excitability of the circuitry.

Air stepping is another pattern seen quite consistently during stretching. Frequently, robust clonic-like movements at the initiation of stretch were replaced with air stepping that varied in intensity and the number of hindlimb segments involved. With more intense air stepping we observed EMG bursts in rectus femoris, but in most cases, despite being rhythmic and frequent air stepping did not result in detectable EMG in the knee muscles. Consistently with previous observation, spasms frequently occurred at the end of a 1 min stretch. Sometimes clonic-like responses had a spasm like appearance, with wider and less consistent peaks within the clonic episode. Early after injury we also observed spasms that were quite subtle in appearance, almost like an isometric contraction-increased EMG responses in the knee muscles of the contralateral limb were apparent but only slight movements in the toes were seen while a static stretch was maintained on the opposite limb.

In this experiment we used a custom designed glove to measure forces during the four major stretches. We found that the highest forces applied during tibialis anterior stretch resulted in torques that averaged around $6 \mathrm{~N} \cdot \mathrm{cm}$, while the lowest torques of $0.8 \mathrm{~N} \cdot \mathrm{cm}$ were applied during the Gastrocnemius stretch. One of the limitations of the force sensors used in the current experiment is that the accuracy of their measurement is somewhat dependent on the precise localization of the center of the sensor to the point of the highest force application. The Tibialis Anterior stretch was perhaps the easiest to achieve a precise positioning of the force sensors, as the index finger sensor was placed on the heel of the rat (a very distinct landmark which was smaller than the sensor itself) and the thumb was placed on the dorsal part of the foot. In addition, end ROM of the ankle plantarflexion is achieved more readily and has a more solid end-feel (resistance) than the other stretching maneuvers which may also help to explain the higher torques achieved during this stretch. While the torques achieved during TA, Gastrocnemius and Hamstring stretches were consistent over time, there was a gradual increase in torques applied during the Quadriceps stretch. As animals recovered from SCI, a greater number of clonic-like contractions occurred, particularly during the quadriceps stretch, which likely contributed to the increase in measured torque over time.

To our knowledge, the current study is the first to report force/torque measurements during therapeutic stretching in any animal model of SCI. However, a few studies have addressed the issue during stretching in human subjects with SCI. Harvey et al. [31], quantified the torque applied during hamstring stretch by twelve different PTs. The range of torque varied substantially with a median in the range of $30-68 \mathrm{~N} \bullet \mathrm{m}$, while some therapists applied over $100 \mathrm{~N} \bullet \mathrm{m}$. If we compare torques about the hip for human and rat based strictly on body weight (average weight of subjects from Harvey's study $-73 \mathrm{~kg}$, and average weight of the rats in our study $-0.25 \mathrm{~kg}$ ) the ratio would approximately be 290:1. The equivalent range of torques for rats during the hamstring stretch would therefore be $10-23 \mathrm{~N} \cdot \mathrm{cm}$. In this study, the average torque during hamstring stretch was around $3 \mathrm{~N} \bullet \mathrm{cm}$. In another study by Harvey et al. [32], they examined a 4 week stretching intervention focused on the gastrocnemius using a set torque of $7.5 \mathrm{~N} \cdot \mathrm{m}$ while ROM assessment was performed using $10 \mathrm{~N} \cdot \mathrm{m}$ torque. Once again, equivalent torques for rats would be 2.8 and 3.8 $\mathrm{N} \cdot \mathrm{cm}$. The average torque applied during gastrocnemius stretch in the current experiment was $0.8 \mathrm{~N} \cdot \mathrm{cm}$. This comparison has obvious limitations, for example, it is not known if the sensitivity to applied torques in rats is comparable to humans, nonetheless, the torques applied in this study are still well under those reported for human patients for these stretches when scaled for body weight.

In three different rat studies using randomized group assignments, blinded assessments, and objective kinematicbased functional outcomes $[6,7,16]$ we showed that a daily clinically-based hindlimb stretching protocol causes significant locomotor deficits in rats with incomplete T10 spinal cord injuries. Thus, the primary goal of the current study was to document the EMG responses of the rat hindlimb muscles to these stretching maneuvers. However, some secondary observations regarding the effects of stretch on locomotor function were also made. Twice a week stretching of one limb for the first 6 weeks did not result in any overt disruption locomotor function as daily bilateral stretching had done in previous studies $[6,7,16,33]$. There were slight drops in the regularity index after every stretching session due likely to an increase in the number of dorsal steps, however it wasn't until unilateral stretching 
was performed every day (weeks 7 and 8 ) that there was a drop in BBB subscores indicating an overt change in stepping (reduction in toe clearance and increased paw rotation). This pattern of subtle disruption taken together with drops in BBB subscores suggests that the "low" dose of stretching used in this experiment had an effect only on the finer motor control aspects of locomotion. These results help us to better understand the stretching phenomenon. We would speculate that the negative effects of stretching after SCI may be mechanistically related to the modest negative effects of stretching on some aspects of motor performance (isometric strength, muscle strength endurance, isokinetic torque production, etc.) seen in intact human subjects $[8,10$, $11]$.

In conclusion, we have described two distinct EMG responses elicited by hindlimb stretching in rats that are similar to those reported for human subjects: clonic-like responses and spasms. We also determined that the torques used during therapeutic stretching of rat hindlimbs are comparable to or lower than those PTs apply to human patients when scaled simply to body weight. Although it would be ideal to design a similar experiment in patients with SCI in order to directly compare the findings between human and rat, the existing evidence suggests that stretching may have similar effects on the nervous system in humans as it does in rats. Therefore, further investigation of stretching and its effects on motor function in human subjects is warranted.

\section{Compliance with ethical standards}

Conflict of interest The authors declare that they have no conflict of interest.

\section{References}

1. Nas K, Yazmalar L, Sah V, Aydin A, Ones K. Rehabilitation of spinal cord injuries. World J Orthop. 2015;6:8-16.

2. Strommen JA. Management of spasticity from spinal cord dysfunction. Neurol Clin. 2013;31:269-86.

3. Harvey LA, Herbert RD. Muscle stretching for treatment and prevention of contracture in people with spinal cord injury. Spinal Cord. 2002;40:1-9.

4. Williams PE. Use of intermittent stretch in the prevention of serial sarcomere loss in immobilised muscle. Ann Rheum Dis. 1990;49:316-7.

5. Harvey LA, Katalinic OM, Herbert RD, Moseley AM, Lannin NA, Schurr K. Stretch for the treatment and prevention of contracture: an abridged republication of a Cochrane Systematic Review. J Physiother. 2017;63:67-75.

6. Keller AV, Wainwright GN, Shum-Siu A, Prince D, Hoeper A, Martin E, et al. Disruption of locomotion in response to hindlimb muscle stretch at acute and chronic time points after a spinal cord injury in rats. J Neurotrauma. 2016;34:661-70.

7. Keller AV, Rees KM, Prince DC, Morehouse JR, Shum-Siu A, Magnuson DS. Dynamic "range of motion" hindlimb stretching disrupts locomotor function in rats with moderate subacute spinal cord injuries. J Neurotrauma. 2017;34:2086-91.

8. Young W, Elliott S. Acute effects of static stretching, proprioceptive neuromuscular facilitation stretching, and maximum voluntary contractions on explosive force production and jumping performance. Res Q Exerc Sport. 2001;72:273-9.

9. Nelson AG, Kokkonen J, Arnall DA. Acute muscle stretching inhibits muscle strength endurance performance. J Strength Cond Res. 2005;19:338-43.

10. Nelson AG, Guillory IK, Cornwell C, Kokkonen J. Inhibition of maximal voluntary isokinetic torque production following stretching is velocity-specific. J Strength Cond Res. 2001;15:241-6.

11. Nelson AG, Allen JD, Cornwell A, Kokkonen J. Inhibition of maximal voluntary isometric torque production by acute stretching is joint-angle specific. Res Q Exerc Sport. 2001;72:68-70.

12. Avela J, Kyrolainen H, Komi PV. Altered reflex sensitivity after repeated and prolonged passive muscle stretching. J Appl Physiol. 1999;86:1283-91.

13. Cramer JT, Housh TJ, Coburn JW, Beck TW, Johnson GO. Acute effects of static stretching on maximal eccentric torque production in women. J Strength Cond Res. 2006;20:354-8.

14. Harvey LA, Glinsky JA, Katalinic OM, Ben M. Contracture management for people with spinal cord injuries. NeuroRehabilitation. 2011;28:17-20.

15. Magnuson DS, Smith RR, Brown EH, Enzmann G, Angeli C, Quesada PM, et al. Swimming as a model of task-specific locomotor retraining after spinal cord injury in the rat. Neurorehabil Neural Repair. 2009;23:535-45.

16. Caudle KL, Atkinson DA, Brown EH, Donaldson K, Seibt E, Chea $\mathrm{T}$, et al. Hindlimb stretching alters locomotor function after spinal cord injury in the adult rat. Neurorehabil Neural Repair. 2015;29:268-77.

17. Bandy WD, Irion JM. The effect of time on static stretch on the flexibility of the hamstring muscles. Phys Ther. 1994;74:845-50. discussion 50-2

18. Kuerzi J, Brown EH, Shum-Siu A, Siu A, Burke D, Morehouse J, et al. Task-specificity vs. ceiling effect: step-training in shallow water after spinal cord injury. Exp Neurol. 2010;224:178-87.

19. Basso DM, Beattie MS, Bresnahan JC. A sensitive and reliable locomotor rating scale for open field testing in rats. J Neurotrauma. 1995;12:1-21.

20. Jonkers BW, Sterk JC, Wouterlood FG. Transcardial perfusion fixation of the CNS by means of a compressed-air-driven device. J Neurosci Methods. 1984;12:141-9.

21. Magnuson DS, Trinder TC, Zhang YP, Burke D, Morassutti DJ, Shields CB. Comparing deficits following excitotoxic and contusion injuries in the thoracic and lumbar spinal cord of the adult rat. Exp Neurol. 1999;156:191-204.

22. Agarwal GC, Gottlieb GL. Oscillation of the human ankle joint in response to applied sinusoidal torque on the foot. J Physiol. 1977;268:151-76.

23. Wallace DM, Ross BH, Thomas CK. Characteristics of lower extremity clonus after human cervical spinal cord injury. J Neurotrauma. 2012;29:915-24.

24. Adams MM, Hicks AL. Spasticity after spinal cord injury. Spinal Cord. 2005;43:577-86.

25. Hultborn H, Malmsten J. Changes in segmental reflexes following chronic spinal cord hemisection in the cat. I. Increased monosynaptic and polysynaptic ventral root discharges. Acta Physiol Scand. 1983;119:405-22.

26. Bose P, Parmer R, Thompson FJ. Velocity-dependent ankle torque in rats after contusion injury of the midthoracic spinal cord: time course. J Neurotrauma. 2002;19:1231-49. 
27. Bennett DJ, Gorassini M, Fouad K, Sanelli L, Han Y, Cheng J. Spasticity in rats with sacral spinal cord injury. J Neurotrauma. 1999;16:69-84.

28. van Gorp S, Deumens R, Leerink M, Nguyen S, Joosten EA, Marsala M. Translation of the rat thoracic contusion model; part 1supraspinally versus spinally mediated pain-like responses and spasticity. Spinal Cord. 2014;52:524-8.

29. Hagbarth KE, Wallin G, Lofstedt L, Aquilonius SM. Muscle spindle activity in alternating tremor of Parkinsonism and in clonus. J Neurol Neurosurg Psychiatry. 1975;38:636-41.

30. Calancie B. Spinal myoclonus after spinal cord injury. J Spinal Cord Med. 2006;29:413-24.
31. Harvey LA, McQuade L, Hawthorne S, Byak A. Quantifying the magnitude of torque physiotherapists apply when stretching the hamstring muscles of people with spinal cord injury. Arch Phys Med Rehabil. 2003;84:1072-5.

32. Harvey LA, Batty J, Crosbie J, Poulter S, Herbert RD. A randomized trial assessing the effects of 4 weeks of daily stretching on ankle mobility in patients with spinal cord injuries. Arch Phys Med Rehabil. 2000;81:1340-7.

33. Caudle KL, Brown EH, Shum-Siu A, Burke DA, Magnuson TS, Voor MJ, et al. Hindlimb immobilization in a wheelchair alters functional recovery following contusive spinal cord injury in the adult rat. Neurorehabil Neural Repair. 2011;25:729-39. 